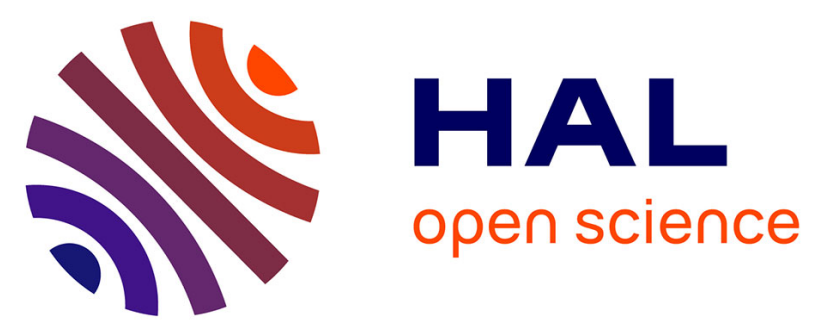

\title{
Structure et propriétés de la matrice des composites WC-W-Ni-Cu-Zn-Si renforcés avec des diamants synthétiques
}

H. Colan, V. Constantinescu, R. Orban

\section{- To cite this version:}

H. Colan, V. Constantinescu, R. Orban. Structure et propriétés de la matrice des composites WC-W$\mathrm{Ni}-\mathrm{Cu}-\mathrm{Zn}-\mathrm{Si}$ renforcés avec des diamants synthétiques. Journal de Physique IV Proceedings, 1993, 03 (C7), pp.C7-1805-C7-1810. 10.1051/jp4:19937286 . jpa-00251928

\section{HAL Id: jpa-00251928 https://hal.science/jpa-00251928}

Submitted on 1 Jan 1993

HAL is a multi-disciplinary open access archive for the deposit and dissemination of scientific research documents, whether they are published or not. The documents may come from teaching and research institutions in France or abroad, or from public or private research centers.
L'archive ouverte pluridisciplinaire HAL, est destinée au dépôt et à la diffusion de documents scientifiques de niveau recherche, publiés ou non, émanant des établissements d'enseignement et de recherche français ou étrangers, des laboratoires publics ou privés. 


\title{
Structure et propriétés de la matrice des composites WC-W-Ni-Cu- Zn-Si renforcés avec des diamants synthétiques
}

\author{
H. COLAN, V. CONSTANTINESCU et R. ORBAN \\ Université Technique de Cluj-Napoca, Cluj-Napoca, Romania
}

\begin{abstract}
- Abstract -
Although seems very attractive for economical reasons, the natural by synthectic diamonds replacement in the metal-diamond composites elaboration comes up against theirs less thermochemical stability. In order to avoid the possible reinforcement degradation and poor matrixto-reinforcement bonding resulting from extensive interfacial reactions, processing temperatures below of about $1050^{\circ} \mathrm{C}$ are required. On effect, the $\mathrm{Cu}-\mathrm{Sn}-\mathrm{Ni}$ binding alloys utilised in the metal-natural diamond composites elaboration by consecrated method - sintering by infiltration of loose mixture of powders, having processing temperatures of about $1150-1225^{\circ} \mathrm{C}$, becomes unsuitable.
\end{abstract}

The author's investigations have found as adequate for this purpose certain $\mathrm{Cu}-\mathrm{Zn}-\mathrm{Si}$ alloys $(65 \ldots 70)-(25 \ldots 30)-$ $(2.5 .3 .5) \%$ having the liquidus temperatures $851 . .870^{\circ} \mathrm{C}$, as well as a good wettability of solids. Also, certain WC-W-Ni $(30 \ldots 90)-(0 \ldots 40)-(3 \ldots 16) \%$ mixtures of powders ware found as appropriates. Solving $\mathrm{Ni}$ and, partial, both $W$ and $W C$, the above mentioned infiltrating alloys act as a liquid phase and make possible the sintering process at temperatures of about $1025^{\circ} \mathrm{C}$, leading to a good mechanical encapsulation of diamond grains, a fine structure of metal matrix and, consequently, to mechanical properties of the matrix comparable with those obtained with $\mathrm{Cu}-\mathrm{Sn}-\mathrm{Ni}$ binding alloys at the processing temperatures upper with about $200^{\circ} \mathrm{C}$.

Pour la fabrication des outils à diamants, en utilisant la méthode du frittage par infiltration des mélanges de poudres librement versés, l'alliage d'infiltration - le liant - forme avec les composants du mélange de poudres la matrice métallique complexe destinée au sertissage des principaux éléments actifs des outils - les granules de diamant - ainsi qu'à la prise des sollicitations mécaniques, thermiques, de corrosion et érosion pendant le fonctionnement.

A part la fluidité, le liant fondu doit avoir une capacité élevée d'humectage des granules de poudre, tandis que les propriétés 
mécaniques, la résistance à l'usure abrasive et d'érosion, la conductivité thermique nécéssaire pour la dissipation de la chaleur dégagée dans la zone des diamants doivent être aussi grands que possible.

La température liquidus du liant et par conséquent la température d'infiltration doivent être les plus basses que possible afin que les diamants naturels et surtout ceux synthétiques ne soient pas affectés qualitativement ou dégradés. En utilisant des liants avec la température d'infiltration au-dessus de $1100^{\circ} \mathrm{C}$, l'intégrité physique des granules de diamant est affectée par grafitisation à la surface ou même intérieure, fissuration, pertes de masse, modifications granulométriques etc.

Tableau 1. Composition des liants (\%)

\begin{tabular}{||l|l|c|c|c|c|c|c||}
\hline \hline Liant & Cu & Sn & Zn & Ni & Si & $\begin{array}{c}\text { Fe-Mo } \\
(60 \% M 0)\end{array}$ & $\begin{array}{c}\text { Fe-Mn } \\
(85 \% \text { Mn })\end{array}$ \\
\hline$L_{1}$ & 79 & 14 & - & - & 1 & 3 & 3 \\
$L_{2}$ & 75 & 6 & - & 10 & 1 & 2 & 5 \\
$L_{3}$ & 62 & 7 & - & 22 & 1 & 2 & 5 \\
\hline E & 67 & - & 30 & - & 3 & - & - \\
$F$ & 71,5 & - & 25 & - & 3,5 & - & - \\
$G$ & 70,5 & 1,5 & 25 & - & 3 & - & - \\
$H$ & 70 & 1 & 24 & - & 3 & 2 & - \\
I & 69 & 1 & 26 & - & 2,5 & 1 & 0,5 \\
\hline
\end{tabular}

La composition des liants réalisée par suite des recherches et fabriqués à Diarom - Bucarest est présentée dans le tableau 1. En utilisant des liants $\mathrm{Cu}-\mathrm{Sn}-\mathrm{Ni}-(\mathrm{Mn}-\mathrm{Si}-\mathrm{Mo}-\mathrm{Fe})$ - des bronzes complexes à étain, les temperatures d'infiltration sont au-

Tableau 2. Intervalle de solidification et température $d^{\prime}$ infiltration des liants $\left({ }^{\circ} \mathrm{C}\right)$

\begin{tabular}{|c|c|c|c|}
\hline Liant & $t_{\text {liquidus }}$ & $t_{\text {solidus }}$ & $t_{\text {infilitation }}$ \\
\hline$L_{1}$ & 892 & 773 & 1150 \\
$I_{2}$ & 1025 & 925 & 1180 \\
$L_{3}$ & 1046 & 869 & 1215 \\
\hline$E$ & 851 & 833 & $1000-1050$ \\
$F$ & 870 & 841 & $1000-1050$ \\
\hline
\end{tabular}

dessus de $1150{ }^{\circ} \mathrm{C}$ (tab.2); les liants $\mathrm{E}, \mathrm{F}, \mathrm{G}, \mathrm{H}, \mathrm{I}$ - des laitons à silicium ou complexes Cu-Zn-Si-(Mo-Sn-Mn-Fe)-ont les températures liquidus beaucoup plus réduites, un intervalle de solidification réduit et une bonne fluidité. La température d'infiltration baisse à $1000-1050^{\circ} \mathrm{C}$.

Les caractéristiques mécaniques sont très rapprochées de celles des liants L (bronzes); la résistance à la traction et à l'usure sont plus élevées (tab.3). 
Tableau 3. Propriétés mécaniques des liants

\begin{tabular}{||c|c|c|c|c|c|c|c||}
\hline \hline Liant & $\begin{array}{c}\mathrm{R}_{\mathrm{m}} \\
\mathrm{MPa}\end{array}$ & $\begin{array}{c}\mathrm{R}_{\text {comp }} \\
\mathrm{MPa}\end{array}$ & $\begin{array}{c}\mathrm{R}_{\text {flex. }} \\
\mathrm{MPa}\end{array}$ & $\begin{array}{c}\mathrm{E} \\
\mathrm{MPa}\end{array}$ & $\begin{array}{c}\mathrm{K} \\
\mathrm{J} / \mathrm{cm}^{2}\end{array}$ & $\mathrm{HRB}$ & $\begin{array}{c}\mathrm{RUA} \\
\mathrm{J} / \mathrm{mm}^{3}\end{array}$ \\
\hline $\mathrm{L}_{1}$ & $250-270$ & $1030-1080$ & $530-560$ & 108000 & $22-37$ & $87-91$ & 870 \\
\hline $\mathrm{E}$ & $440-460$ & $1020-1080$ & $590-610$ & 108000 & $20-23$ & $88-91$ & 920 \\
$\mathrm{~F}$ & $330-380$ & $1000-1050$ & $590-620$ & 104000 & $27-36$ & $93-97$ & 940 \\
\hline
\end{tabular}

La microstructure des laitons $\mathrm{E}$ (équivalent de $\mathrm{Zn} 47 \%$ ) et $\mathrm{F}$ (équivalent de $\mathrm{Zn} 45,6$ \%) est $\alpha+\beta^{\prime}$ y compris aussi l'eutectoïde $\alpha+\tau$ du système $\mathrm{Cu}-\mathrm{Si}$. Dans les alliages $\mathrm{Cu}-\mathrm{Zn}-\mathrm{Si}$, le zinc déplace à

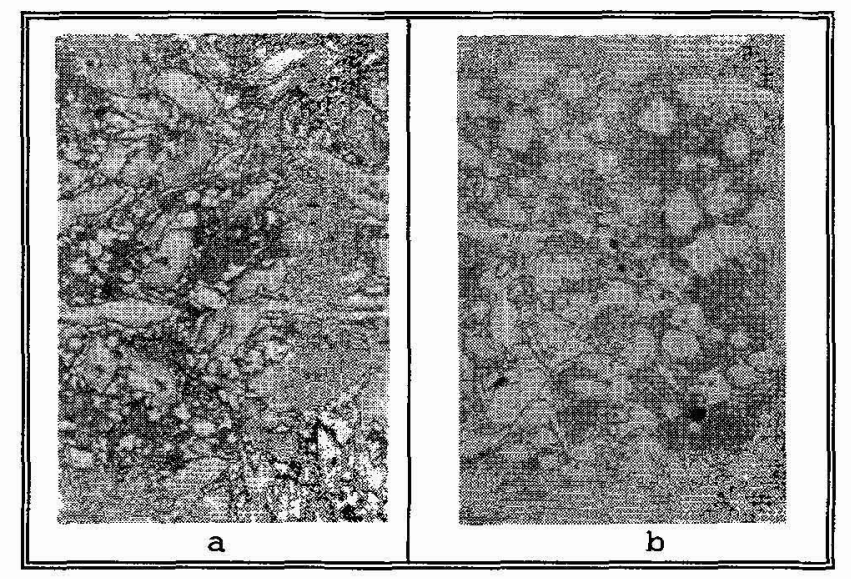

Fig.1 Liants $a-E ; b-H \quad 200: 1$

gauche les lignes du diagramme Cu-Si, en diminuant la solubilité du silicium dans le cuivre et le contenu en silicium de l'eutectoïde. De telle sorte que celui-ci est possible d'apparaitre dans la structure au bas contenus de silicium, les aliages se maintenant dans le système Cu-Si. Ainsi,leur structure sera $\alpha$, eutectoïde $\alpha+\tau$ (CuSizn) et $B^{\prime}$ (Cuznsi) (fig.1).

Le silicium agit comme désoxydant, réduit l'intervalle de solidification du laiton, améliore la fluidité et la contraction. Il est limité à 3-4 pour éviter 1'apparition de la phase $\tau\left(\mathrm{Cu}_{5} \mathrm{Si}\right)$ libre, fragile, au joints des grains.

Le fer, le manganèse et le molybdène améliorent les propriétés mécaniques grâce à une structure plus fine (centres supplémentaires de cristallisation, séparations secondaires de $\mathrm{FeZn}_{7}$ dans les phases $B^{\prime}$ et $\alpha$ ). Le manganèse réduit aussi la solubilité du silicium dans le cuivre, huit fois plus fort que le zinc.

Le mélange de poudres doit comprendre des éléments qui forment des solutions solides avec le liant ou des nouveaux constituants qui améliorent les propriétés. De plus, pour l'alliage - matrice il est 
Tableau 4. Composition des mélanges des poudres $(\%)$

\begin{tabular}{|c|c|c|c|c|}
\hline Mélange & WC & w & Ni & Autres \\
\hline$A_{1}$ & 83 & - & 17 & \\
$A_{2}$ & 84 & - & 16 & \\
$A_{3}$ & 92 & - & 8 & \\
$A_{4}$ & 55 & 40 & 5 & 1,5 flux \\
$A_{5}$ & 72 & 21,5 & 5 & 1,5 flux \\
$A_{6}$ & 29 & 67 & 2,5 & \\
$A_{7}$ & 89 & 5 & 6 & 15 Cu \\
$A_{8}$ & - & 80 & 5 & \\
$A_{9}$ & - & 86 & 14 & \\
\hline
\end{tabular}

nécéssaire une capacité d'humectage des granules de diamant. Les mélanges de poudre ont été $\mathrm{WC}-\mathrm{Ni}$, WC-W-Ni et $\mathrm{W}-\mathrm{Ni}-(\mathrm{Cu})(\mathrm{tab} .4)$, la température d'infiltration $1050 \mathrm{C}$, les propriétés mécaniques des

Tableau 5. Propriétês mécaniques des matrices

\begin{tabular}{|c|c|c|c|c|}
\hline Matrice & $\begin{array}{l}R_{\text {flex }} \cdot \\
\mathrm{MPa} \\
\end{array}$ & $\begin{array}{r}\mathrm{R}_{\text {comp }} \\
\mathrm{MPa} \\
\end{array}$ & HRC & $\begin{array}{c}\mathrm{RUA} \\
\mathrm{J} / \mathrm{mm}^{3}\end{array}$ \\
\hline $\begin{array}{l}\mathrm{A}_{1}, \mathrm{E} \\
\mathrm{A}_{1}, \mathrm{~L}_{3}\end{array}$ & $\begin{array}{l}520-590 \\
540-600 \\
\end{array}$ & $\begin{array}{r}980-1020 \\
1150-1300 \\
\end{array}$ & $\begin{array}{l}38-43 \\
35-46 \\
\end{array}$ & $\begin{array}{c}7870 \\
7480-8260 \\
\end{array}$ \\
\hline $\begin{array}{l}\mathrm{A}_{3}, \mathrm{~F} \\
\mathrm{~A}_{3}, \mathrm{~L}_{3} \\
\end{array}$ & $\begin{array}{l}340-430 \\
520-620 \\
\end{array}$ & $\begin{array}{r}850-880 \\
1200-1300 \\
\end{array}$ & $\begin{array}{l}41-46 \\
42-50 \\
\end{array}$ & $\begin{array}{c}6210 \\
6090-6740 \\
\end{array}$ \\
\hline $\begin{array}{l}\mathrm{A}_{4}, \mathrm{E} \\
\mathrm{A}_{4}, \mathrm{~F} \\
\mathrm{~A}_{4}, \mathrm{~L}_{1}\end{array}$ & $\begin{array}{l}500-570 \\
460-510 \\
480-520 \\
\end{array}$ & $\begin{array}{l}790-900 \\
830-1000 \\
800-880 \\
\end{array}$ & $\begin{array}{l}32-42 \\
36-42 \\
26-41 \\
\end{array}$ & $\begin{array}{c}3980 \\
4050 \\
3740-4130 \\
\end{array}$ \\
\hline $\begin{array}{l}\mathrm{A}_{5}, \mathrm{E} \\
\mathrm{A}_{5}, \mathrm{~F} \\
\mathrm{~A}_{5}, \mathrm{~L}_{1}\end{array}$ & $\begin{array}{l}280-320 \\
450-540 \\
300-380 \\
\end{array}$ & $\begin{array}{r}790-880 \\
880-930 \\
1000-1100 \\
\end{array}$ & $\begin{array}{l}34-40 \\
35-44 \\
32-45 \\
\end{array}$ & $\begin{array}{c}4190 \\
4310 \\
4000-4530 \\
\end{array}$ \\
\hline $\begin{array}{l}\mathrm{A}_{8}, \mathrm{~F} \\
\mathrm{~A}_{8}, \mathrm{~L}_{1}\end{array}$ & $\begin{array}{l}510-550 \\
500-550 \\
\end{array}$ & $\begin{array}{l}600-640 \\
650-700 \\
\end{array}$ & $\begin{array}{l}22-27 \\
16-22(28) \\
\end{array}$ & - \\
\hline
\end{tabular}

matrices obtenues en étant appropriées (tab.5). Ont été fabriqués des outils de forage à performances comparables à celles obtenues avec des liants $\mathrm{Cu}-5 \mathrm{n}-\mathrm{Ni}$ et après le dessertissage on a constaté une intégrité sensiblement meilleure des diamants récupérés.

ont été effectuées des sections métallographiques longitudinales dans les échantillons cylindriques infiltrés (matrices) dans la troisième partie extérieure du rayon, à trois endroits sur la hauteur du cylindre: supérieur, moyen, inférieur pour mettre en évidence les eventuelles non-remplissages ou porosités et ségrégations. Attaque chimique à clorure ferrique.

Par l'infiltration et la réalisation de la matrice, la dissolution du nickel dans l'alliage Cu-Zn-Si donne naissance à une solution solide complexe, avec la présence de la phase $\mathrm{Ni}_{2} \mathrm{Si}$, la solubilité 
de laquelle varie fortement avec la température (possibilité de durcissement par précipitation, en augmentant la résistance à l'usure). Le nickel améliore les propriétés mécaniques: augmente la dureté de la phase $\alpha$ (un peu) et de la phase $\beta^{\prime}$ (beaucoup).

Tableau 6. La structure des matrices

\begin{tabular}{|c|c|c|}
\hline $\begin{array}{c}\text { Mélange de } \\
\text { poudres }\end{array}$ & Matrice & \multicolumn{1}{c|}{ Microstructure } \\
\hline WC-Ni & $\left(A_{1}, A_{2}, A_{3}\right)-(E, F)$ & WC+ $\alpha+$ teutectoïde $(\alpha+\tau)+B^{\prime}$ \\
\hline WC-W-Ni & $\begin{array}{l}\left(A_{4}, A_{5}, A_{6}, A_{7}\right)-(E, F) \\
\left(A_{4}, A_{5}, A_{5}\right)-(G, H, I)\end{array}$ & $\begin{array}{l}\text { WC+W+o+eutectoidde }(\alpha+\tau)+B^{\prime} \\
\text { Idem. Eutectoïde trés } \\
\text { fin (H) et fin (I) dû au } \\
\text { FeMo }\end{array}$ \\
\hline W-Ni-(Cu) & $\left(A_{8}, A_{9}\right)-(E, F)$ & W+ $\alpha+$ teutectoïde $(\alpha+\tau)+B^{\prime}$ \\
\hline
\end{tabular}

figures 2 et 3 . On peut observer que l'eutectoïd le plus fin qui lie les grains de WC et $W$ est obtenu avec des liants $G, H, I$ (surtout H), alliages Cu-Zn-Si complexes, grâce à la présence du fer et du molibdène.

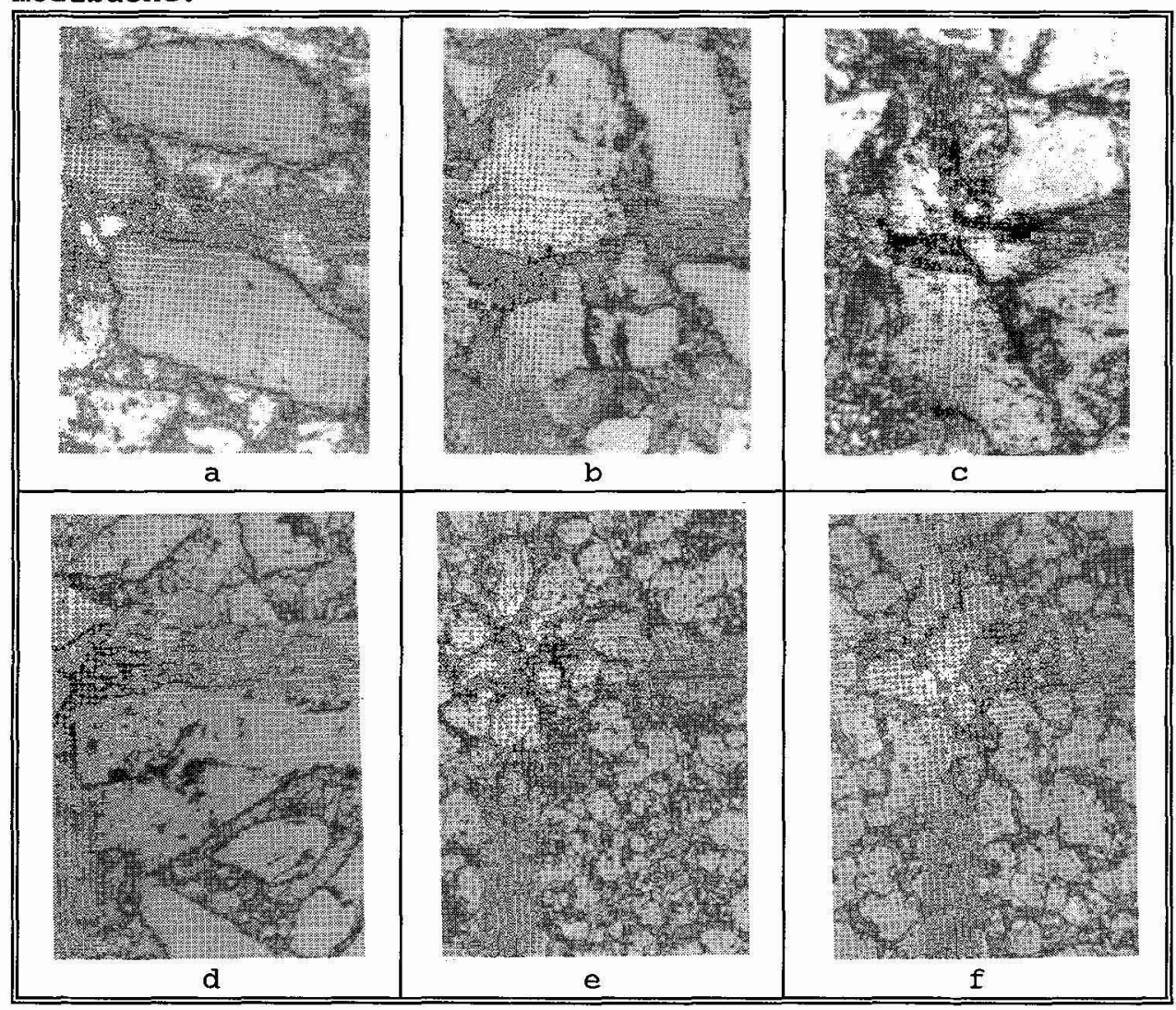

Fig.2 Matrices a $-A_{1} E ; b-A_{1} F ; C-A_{7} E ; d-A_{7} F ; e-A_{8} E ; f-A_{8} F \quad 200: 1$ 
Certaines porosités apparaissent dans les matrices utilisant les mélanges $A_{1}, A_{3}$ à WC (remplissage incomplet); pour les mélanges à $W C$ et $W$ le phénomène ne se rencontre pas souvent et pour ceux à $W$ (sans WC) les pores n'apparaissent pas.
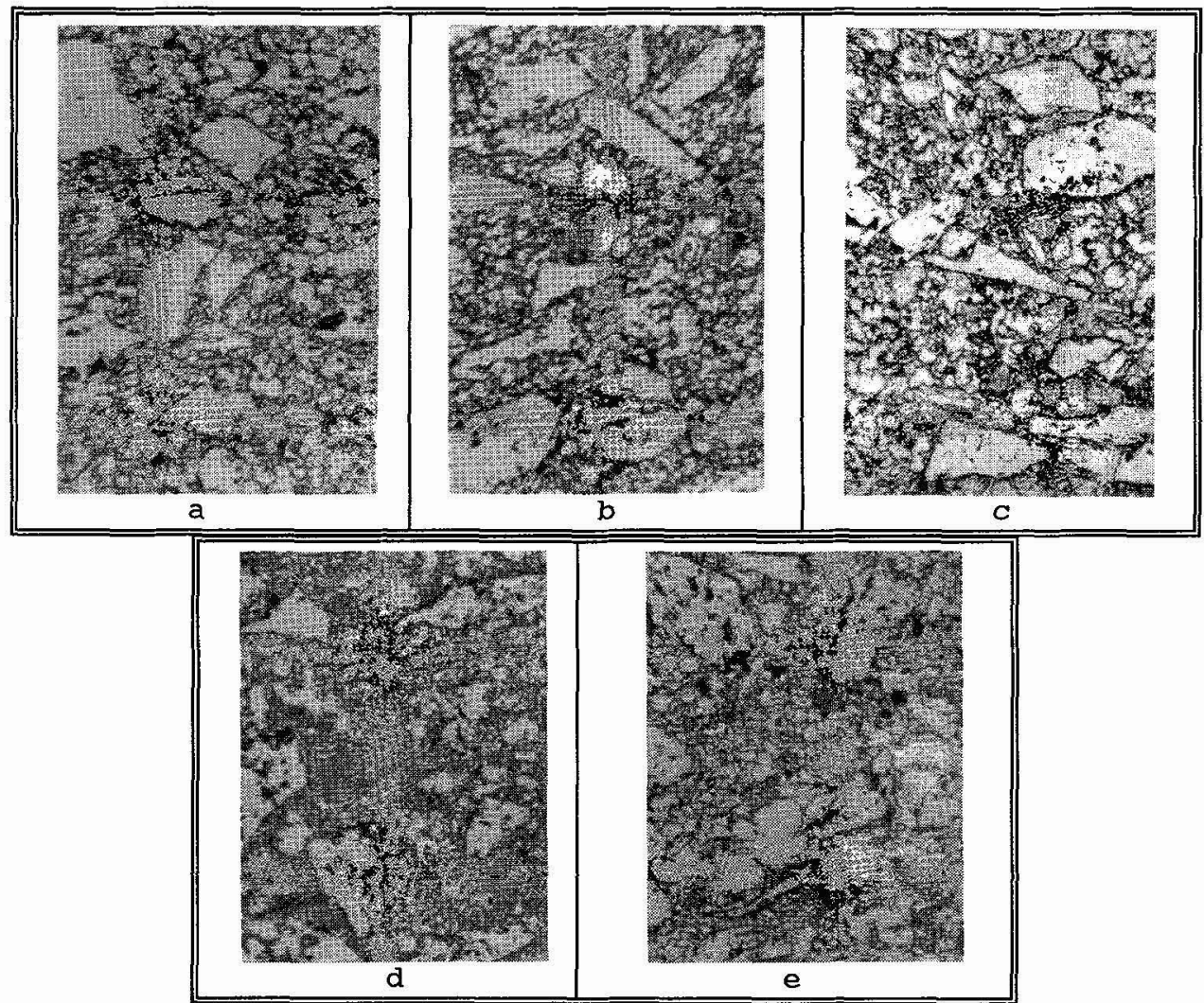

Fig.3 Matrices a $-A_{4} E ; b-A_{4} F ; c-A_{4} G ; d-A_{4} H: e-A_{4} I \quad 200: 1$

Ont été élaborés aussi des étalons pour l'analyse chimique rapide par fluorescence des rayons $X$ des liants, de la matrice et des matières premières métalliques nécéssaires à la fabrication des outils de forage à diamants.

\section{BIBLIOGRAPHIE}

1.H.Colan, V.Constantinescu, R.Orban. Cercetări asupra structurii şi proprietăţilor aliajelor cu-zn-si utilizate ca lianţi pentru sinterizarea prin infiltrare a materialelor compozite cu diamante sintetice. A III-a Conferinţă naţională de metalurgia pulberilor. Cluj-Napoca 1988. Vol.I, p.171-177.

2.H.Colan, V.Constantinescu, R. Orban Materiale compozite WC-W-Ni$\mathrm{Cu}-\mathrm{zn}-\mathrm{Si}$ pentru scule cu rezistenţă Conferinţa naţională de metalurgia ridicată la uzare. A III-a Vol.I. p.165-170. pulberilor. Cluj-Napoca 1988, 